\title{
Antibiotics Misuse and Factors Leading to Its' Abuse in Kurdistan Region
}

\begin{abstract}
Kemal M. Surji PhD, RRT
Hawler Medical University,College of Pharmacy

Abstract

Antimicrobial consumption is a major risk factor for antibiotic resistance and a routine practice in Kurdistan Region. Purchase of antibiotics without prescription by patients is a common practice by self-medication. Similarly, the majority of the people do not adhere to complete course of prescribed antibiotics because they often feel well after taking a few tablets. Unnecessary antibiotic use for viral illness is typical and will lead to increasing rates of antibiotic resistance. Self-medication pervasiveness with antibiotic in Kurdistan is disturbingly high, the unregulated practice of prescribing practitioners and no enforcement of the laws that prevent dispensing without prescription of antibiotics in community pharmacies contribute to antibiotic overuse. Furthermore, lack of education, patients' expectations, past experience, and economic incentives, diagnostic uncertainty, Poor drug quality, unsanitary conditions accounting for the spread of resistant bacteria and most importantly the political corruption and inadequate surveillance are factors for reform in health practice in Kurdistan. Consequently, it is imperative to implement multifaceted interventions to reduce overuse of antibiotics. The recommended interventions are medical and public education, auditing and limiting antibiotic choices, develop prescription guidelines, emphasizing on quality compliance, monitoring manufactured and imported antimicrobial medications, improved public sanitation and hygienic practices, development of a proper hospital infection control procedures. Additionally, transparency of healthcare budget, expenditure and appoint qualified individuals based on expertise in the health system and vitally important the implementation of rapid testing for an antibiogram will enhance the reduction of antibiotic misuse in the region.
\end{abstract}

Keywords: Antimicrobial resistance, antibiotics, antibiogram, prescription, self-medication, misuse, overuse

DOI: $\quad 10.7176 / \mathrm{JHMN} / 24-2016-05$

\section{Introduction}

Current antibiotic consumption is well-known risk factors for microbial resistance. Despite the acknowledgment of this fact, misuse and illogical consumption of antibiotics is a routine practice in Kurdistan Region, where unrestricted access to antibiotics provokes the pervasiveness of infectious disease affliction.

Antibiotic medications are one of the most frequently sold medications in Kurdistan. According to Gyssens 2001, the overuse and misuse of antimicrobial drugs result in bacterial resistance, adverse effect and economic encumbrance on region's health system. This illogical utilization of antibiotics occurs from economic elements, health policies, and doctors' lack of concern regarding drug resistance, the sale and marketing of antibiotics without prescription (Metlay et al., 1998).

In Kurdistan, anyone can visit a community pharmacy and purchase any pharmaceutical product as he or she would at a hypermarket. Similar to most developing countries, having a lawful prescription is not always required for receiving prescription-only drugs. Except narcotics and major tranquilizers, people can buy any drug, including antibiotics, without a prescription.

The majority of the populations have an understanding that antibiotics are effective for the common cold. Mostly people of developing countries believe that even for minor common cold and flu-like symptoms antibiotics are the best choice and consume by self-medications. A study by Edwards et al. in 2002, displays that majority of the people do not adhere to complete course of prescribed antibiotics because they feel better after taking a few tablets.

Self-medication pervasiveness with antibiotic in Kurdistan is disturbingly high, the unregulated practice of prescribing practitioners and no enforcement of the laws that prevent dispensing without prescription of antibiotics in community pharmacies are factors for reform in health practice in Kurdistan.

\section{Objectives of the article}

The aim is not only to reduce the number of antibiotics but also to encourage the use of antibiotics in a logical manner. Antimicrobials should only be prescribed to patients with an evidenced-based diagnosis for bacterial and related infections for the purpose of benefit from the treatment. The following are the specific objectives considered in the study:

- To recognize the factors leading to overuse of antibiotics

- Acknowledgment of developing bacterial resistance by misuse of antimicrobials

- Identify measures that would possibly suppress antibiotic resistance 


\section{Problem Statement}

Overuse and misuse of antibiotics are a global concern. Consequently, the extensive use of antimicrobials places the society at a high risk of antibiotic resistance. Unregulated sale and self-treatment have created the problem of multidrug resistance recently causing a great public health concern. Similarly, Bacterial resistance created greatest economic, social and political repercussions. The rate of antimicrobial resistant is intensely on the rise; therefore, if the health related authorities ignore this severe health issue then society will be facing the ultimate health challenge of the lifetime. The task is to identify the factors leading to overuse of antibiotics then control and monitor the sale and distribution of medication to slow the rate at which resistance develops and spreads (WHO 2000).

In Kurdistan region, there is illiteracy regarding medications and especially bacterial infection versus viral infections. Careless practitioners prescribe unnecessary drugs for the purpose of patients' satisfaction, economic gain or diagnostic uncertainty. In various public and private settings existence of unsanitary conditions are the reasons for contagious diseases requiring overuse of broad-spectrum antibiotics. Astonishingly instead of correcting sanitation issues they prescribe antimicrobial drugs! Most importantly the issue of corruption hinders many facets of enhancement in the healthcare system in the region.

\section{The Significance of the Subject}

Antibiotic resistance is an important health problem that impends health care and creates a national challenge for public health interventions. Kurdistan regions predisposition to many factors that ultimately results in overuse of the antimicrobials. Sanitation problems in health facilities, hygienic issues by health practitioners in handling patient cases including lack of hand washing between patients and disinfecting equipment are the major cause of spreading infectious diseases. Therefore, antibiotics are used to combat community-acquired infections leading to overuse and development of bacterial resistance.

If the problem of overprescribing antibiotics is not immediately dealt with, World Health Organization (WHO) 2012 warns that a simple infection may become uncontrollable and potentially lethal. According to United Kingdom Department of Health 2012, Practitioners need to provide strong evidence-based guidance on antibiotics applicable use. Moreover, Davies et al. 2013 stated that due to loss of battle against the infectious disease, antibiotics may not be effective in the future.

The concerns of bacterial resistance cannot be overlooked as it is the public health crises of near future. Significant attention including economic, political and social matters is imperative in combating this inevitable health catastrophe.

\section{Factual Information}

\section{The factors leading to misuse of antibiotics and its contribution to bacterial resistance in the region are:}

- Knowledge, attitude and behavior toward the use of antibiotics

- Role of prescriber and amateur health practitioners in prescribing antibiotics

- Patient expectations and patients' past experience

- Economic enticements

- Diagnostic uncertainty

- Poor drug quality

- Unsanitary conditions accounting for spread of resistant bacteria

- Political Corruption and Inadequate surveillance

\section{Knowledge, attitude and behavior toward the use of antibiotics}

Education is the key to understanding the advantage and disadvantage of antimicrobial agents. Therefore, Patients' lack of awareness and previous experience contribute to increased demand for antibiotics. Many patients take antibiotics for upper respiratory infections caused by viruses, and since the infection is self-limiting the treatment is considered as effective. Populations with lower levels of educational appear to have the highest fallacies about antibiotic use for viral versus bacterial respiratory infections (Mainous et al. 1997).

According to several pharmacists in Erbil city of Kurdistan, patients approach pharmacists and request amoxicillin, as one of the furthermost prevalent drug in the region, because they have a minor sore throat. These patients believe that amoxicillin is the drug of choice for cold and flu, and nothing will convince them otherwise. Most patients do not differentiate between bacteria and viruses and consider both as the same agent which is cured by antibiotics. Therefore, the region needs serious public health educational campaign to make awareness regarding health issues and the danger relating to misuse of antibiotics.

Role of prescriber and amateur health practitioners in prescribing antibiotics

There is a numerous unnecessary prescription of antibiotics seen in many developing countries particularly in 
Kurdistan region for cases of acute diarrhea and viral respiratory infections. There are many reasons for prescribing unnecessary antibiotics among which there is no standard laboratory test before prescribing drugs instead the physician assumes it is a bacterial infection. Second, patients demand antibiotics causing pressure on physicians to prescribe antimicrobial medications. Third, to satisfy and retain patients for the future visits, physicians prescribe a bag full of medications including needless antibiotics.

In this region (Kurdistan) similar to some other developing nations, skilled health providers are limited and cannot provide services to the entire inhabitants. According to Pearson (1995), in various developing countries, community health personnel and others with a minimal training treat minor ailments. The qualifications, training and quality of care of community health employees differ from nation to nation. Unqualified people have the least knowledge concerning harmful effects of inappropriate utilization of antibiotic.

In Kurdistan misdiagnosed noninfectious diseases are treated with injectable antibiotics concurrently with oral antibiotics for the vast majority of patients by unskilled practitioners. According to numerous patients, some of these injectable antibiotics were administered by the same syringe and needle previously used on other patients since the same medication was drawn up in the syringe and utilize for several patients simultaneously. For example, according to some medical assistants, as they are allowed to open their own clinic for minor treatments in Kurdistan, the practitioner draws $6 \mathrm{cc}$ of an antibiotic at 2cc per patients then he or she would use that for three different patients without changing the syringe or the needle for that matter. This careless and lack of professional practice will subject patients to more serious contagious infection such as hepatitis, HIV, and other major communicable ailments.

\section{Patient expectations and patients' past experience}

It is well-known fact that patients' expectations for antibiotics affect doctors' behavior in prescribing medication (MacFarlane et al. 1997). Antibiotic purchase without a prescription is a common practice in most developing countries; similarly, in Kurdistan antibiotics are liberally obtainable on request from hospitals, pharmacies, and drug stores. It seems like the use of antibiotics has become public cultural beliefs that there is a drug for every symptom and antibiotics can heal many diseases, including digestive disorder, muscle ache, and headaches. It is also strongly believed that injections are more powerful than pills. Therefore, according to Haak and Hardon (1998), the abuse of antibiotics often becomes incorporated into the indigenous culture.

Poor patient compliance in Kurdistan is another reason for antimicrobial misuse. Physician interactions with the patient are habitually insufficient. The visits also lack the required quality. Inadequate interaction leads to poor communication which is specifically responsible for noncompliance of patients with the antibiotic treatments. Second, because patients are unlikely to return for follow-up visits and incur extra expense, therefore, they most likely self-medicate by repurchasing the same antibiotics without consulting the health care provider and also recommend that to other relatives.

Illiteracy is another issue in antibiotic or medication misuse. Most patients may be unable to read medicine labels and the complexities of package insert accompany each medication that requires a well-educated individual to comprehend the material. Finally, due to the high cost of some medications many indigent patients do not purchase the complete treatments rather buy partial regimens whenever possible and discontinue usage when signs and symptoms of disorder disappear without knowing that the pathogen is not being completely eradicated.

\section{Economic enticements}

Economic factors contributing to over-prescription are time pressure and provide utmost patient satisfaction to retain patients in the future. For example, if patients are not prescribed a certain amount of medication, most likely the patient will not visit that particular doctor in the future.

Years of habitual prescribing by physicians or taking antibiotics for viral respiratory infections have created an economic cycle of supply and demand that supports behaviors in promoting antibiotic resistance with a detrimental effect on the society. Ending this habitual practice requires rigors public education regarding flaws in the past practices and to convince physicians that patient satisfaction is more about understanding than prescription (Hamm \& Bemben, 1996).

\section{Diagnostic uncertainty}

According to Dowell 1998, Diagnostic uncertainty also contributes to irrational antibiotic use. A practitioner is more likely to prescribe antibiotic just in case there is an infection, and it might be bacteria when they are not certain about a diagnosis.

The rapid test or point of care tests seems to decrease doctors' diagnostic uncertainty and provides valuable information as to the need for antibiotic use. Antibiogram as a point of care predicts prognosis and projected reaction to antibiotic treatment. According to Lior and Butler, 2014, the etiological agent of an infection is not the most vital factor in primary care; however, the prediction and evaluation of the infectious 
disease and potential effect of antibiotic treatment is considered more essential.

\section{Poor Quality of Medication \\ Environmental factors}

Environmental factors in Kurdistan such as lack of proper electricity that has a direct effect on medication storage temperature, and shipment without proper temperature controlled mechanism are responsible for medication quality. The environmental effect on antibiotics such as the temperature of higher than 25 degrees Celsius during shipment or at the storage and left out in the hot sun for a certain period can cause degradation of antibiotics. Drugs are frequently handled by untrained and unskilled workers who may improperly store them.

\section{Substandard medication}

Antibiotic medicines that do not conform to minimum standards are illegal in all countries. However, in the developing countries, the quality of many antibiotics is below standards. In the majority of circumstances, the only indication of substandard medication is a therapeutic failure. Monitoring and controlling laboratories to identify substandard drugs are uncommon, and health workers, distributors, and consumers are often unaware of such analytical surveillances (Okeke, Lamikanra, Edelman, 1999).

\section{Counterfeit Drugs}

Counterfeit drugs include products with little or no active ingredients, or less expensive alternatives have replaced certain ingredients. Some drugs sold in developing countries with misleading labels that do not contain the concentration of active substances as stated. These forged products flourish, regardless of a crackdown by local regulatory agencies to stop their production and distribution (McGregore, 1997).

Counterfeit drugs, like other forged materials, compete favorably in the markets of developing countries. The analytic for quality control facilities available to law enforcement agencies often cannot detect these drugs before they reach the patient. Because of the abundance of generic medicines in unindustrialized countries, a considerable proportion of counterfeit drugs goes undetected (Okeke, Lamikanra, Edelman, 1999).

In Kurdistan as the patient inquire about a medication the pharmacists display several different brand of the same medication having different prices for the patients to choose from without knowing which alternative has the effective active ingredients. Consequently, patients typically choose the least expensive brand. For instance, Lipitor, a cholesterol-lowering drug, will be presented to the patients as Turkish brand, Jordan brand, US brand with the label indicating the same active ingredients (20mg) ranging from 10 US dollars to 80 us dollars and higher for 30 tablets. There is enough evidence that patients have taken medications for several months without improvement in their conditions which indicates that they have taken counterfeit drugs. The authorities must eliminate the practice of medication option based on price causing therapeutic failure through developing more rigorous monitoring system in the region.

\section{Expired Antibiotics}

Expired antibiotics are another issue in developing countries and regions such as Kurdistan. These drugs are distributed as expired or under the new label with the change in manufacture or expiration date.

\section{Adulterated Drugs}

Adulterated drugs have been reported among herbalists in developing countries. Herbal preparations in developing countries are frequently mixed with conventional drugs. As been reported in a study by Huang, Wen, Hsiao, 24 percent of Chinese herbal compounds promoted in Taiwan contained one or more of such adulterants (1997).

\section{Inadequate Hospital Infection Control Practice}

Insufficient Hospital Infection Control Practices in developing countries are undeveloped and often compromised by economic deficits and opposing traditional values (Meers, 1998). The resulting growth of nosocomial pathogens and resistant organisms may be spread to the public. Improper disposal of hospital waste accentuates such spread. Untreated hospital waste in some regions is often dumped into public sewers or thrown into rubbish piles ravaged by scavengers (Okello et al., 1997). Lack of hand washing by healthcare professional and inadequate use of surgical gloves at the time of patient assessments are common practice in most hospitals in Kurdistan. This irresponsible practice leads to spread of infection from one patient to another causing increase length of hospital stay and multi usage of antibiotics that could have been prevented by a simple hygienic practice.

\section{Political Corruption and Inadequate Surveillance}

In unindustrialized countries, political corruption and maladministration of resources, workforces, and 
development programs have created large populations living in miserable scarceness and at high risk for infection (Cornwall, 1997). In Kurdistan region healthcare budget is not transparent and mismanaged, thereby the budget is only partially used for the medical expenses and development of high-quality medical equipment and training. Most public hospitals are in need of serious renovation and laboratories are not sufficient in detecting of many diagnosis. Furthermore, medical professionals are not updated on new medical enhancement. Therefore, the new graduating students are learning from physicians with rudimentary knowledge of the 1960s and 1970s medical practice and intervention for decades. Since there is limited supervision for new graduating doctors, treatments are based on trial and error and patients are considered as laboratory objects.

Health workers are paid poorly, and benefits are nonexistent. Personnel are not hired based on the knowledge but according to whom they know in upper management. Healthcare posts are filled by individuals with lack of management and leadership skills having very limited knowledge of developing programs for improving the health system in the region. Therefore, the public is in danger of the grave situation of developing bacterial resistance to antibiotics due to the immoral and corrupt system. Consequently, individuals with communicable diseases, unable to afford medical treatment, may infect others and spread the resistant strain of contagious bacteria among people in the community.

\section{Strategy for Battling the Antibiotic Misuse and Containment of Antibiotic Resistance}

Many studies have shown the effectiveness of different forms of intervention in supporting a more meaningful use of antibiotics. According to Carl Llor and Lars Bjerrum 2014, the most successful interventions to improve antibiotic prescribing are displayed as multifaceted interventions uniting physician, healthcare providers, patient and public education in diverse settings. Collaborative educational conferences displayed more effective than didactic lectures, but levels of improvement are shown to be limited.

According to Couper (1997), World Health Organization (WHO) has recommended great advice for safeguarding proper medication use to adapt for battling the growth of community-acquired antibiotic resistance in developing countries. In Kurdistan region, the exploitation of antibiotics by health-care professionals, amateur practitioners, and the public can be relieved by auditing antibiotics, limiting antibiotic choice, developing prescription guidelines and stressing the importance of continuing medical and public education. The quality of antibiotics can be enhanced by accentuating quality compliance and monitoring manufactured and imported antimicrobial medications. Therefore, such restructurings will limit substandard drugs that are degraded, counterfeit, and adulterated.

Spreading of bacterial resistance in the region can be inhibited by improved public sanitation and hygienic practices along with the development of a strict hospital infection control procedures. Finally, formulate strategies to warrant the implementation and adaptation of these recommendations under recent challenging economic and political circumstances. Antimicrobial resistances will be an important health issue in Kurdistan region and continue to deteriorate unless educative actions are instituted. 
Table 1. Antibiotic Misuse Factors, Recommendations, and possible results

Healthcare Professionals

Amateur practitioners

Public demand
1. Auditing antibiotics,

2. Limiting antibiotic choice,

3. Developing prescription Guidelines

4. Continuing medical and public education

1. Emphasizing on quality compliance

2. monitoring manufactured and imported antimicrobial medications

- counterfeit

- $\quad$ adulterated

Unsanitary conditions

Political Corruption Inadequate Surveillance
1. Improved public sanitation and hygienic practices

2. Development of a strict hospital infection control procedures

1. Transparency of budget and expenditure

2. Appoint qualified individuals based on expertise

3. Decent salary for healthcare providers

4. New graduating doctors to be supervised while on duty

5. Rapid, low-cost, local laboratory facility for an antibiogram
Reduction in antibiotic prescription and misuse

Limit substandard drugs that are degraded, counterfeit, and adulterated.

Restrict the need for overuse of antibiotics

Hospital renovation Laboratories for diagnostic procedures

Develop plan \& programs

Enhance healthcare system

Develop self-confident \&

well-trained physicians

Instant identification of need for the appropriate antimicrobials

Source: Author

\section{Discussion}

Unregulated access to antibiotics and illogical consumption of such medications is a common practice and the major cause of recurrent infectious disease in the region. In Kurdistan, unnecessary antibiotics are often prescribed; such that doctors prescribe antimicrobials to pediatric patients knowingly the infection is the most likely viral origin. Inappropriate Self-medication has exacerbated the misuse of the antibiotics. It is a well-known fact in the absence of supervision by healthcare professionals; most of our society consumes nonprescription antibiotics for treating viral respiratory infections such as common cold. The unregulated medications will lead to the surge of microbial resistance causing antibiotic ineffectiveness producing harm to the patients and the society. According to a study by Goossens et al. 2005, the regions with the most antibiotic use have the top rate of antibiotic resistance. Therefore, antibiotic ineffectiveness is a major factor for increased morbidity and premature mortality. The infection caused by antibiotic resistance organisms accumulate much higher expenditure and extended hospital stay as well as second and third-line drugs requirement with possibly less efficient, and more toxic reactions (Levy 2005). It is evident that the developments of the new generation of antimicrobials are limited; therefore, the restrictive and applicable utilization of antibiotics to safeguard the accessibility and effectiveness of antibiotics against bacterial infections are of vital concern.

As indicated in the study the factors influencing the spread of antibiotic resistance are numerous and interrelated. The multifactorial causes are illogical, incongruous, high intake, and reckless consumption of antibiotics as well as patients noncompliance with dosage as prescribed. Doctors frequently skip the supporting diagnosis to confirm the bacterial infections, and unswervingly prescribe antibiotics whenever they suspect infections. Patients regard antibiotics as magical medicines that treat all medical problems especially when the physicians prescribe them on habitual basis rather than diagnostic confirmation. Patients tend to ignore medical and professional visits and choose to self-medicate for common diseases, in particular, nonprescription antibiotics. Furthermore, patients have a habit of duplicating their previous prescriptions for future use or sharing with family and friends for the similar medical conditions.

Antibiotics are effortlessly acquired at a low price without prescription in the majority of community pharmacies, drug stores or even in roadside stands. Most common antibiotics are sold at comparatively 
inexpensive in this region to those of western countries with lower quality, purity, and dubious authenticity. Accordingly in Kurdistan region of Iraq, antibiotic acquisition and consumption are relatively uncontrolled, and self-medication with antibiotics such as amoxicillin, ampicillin and related antimicrobials for the treatment of minor diseases such as viral infections of upper respiratory tract, common cold and cough is most common.

A booming market for the antibiotic has expanded regionally as such the substandard antibiotics have dominated the market. A substandard antibiotic most likely causes therapeutic failure due to the impurity, lower dosage, and absence of active ingredient. Consequently, these adulterated medications induce antimicrobial resistance, increase morbidity, mortality and significant economic burden on regional government. According to Issack MI (2001) subscriber might believe that substandard narrow-spectrum antibiotics are not effective, hence, needlessly prescribe a newer broad-spectrum antibiotics as their first-line treatment for many infections.

It is imperative that healthcare authorities in the region to implement and enhance a surveillance program to guarantee the drug-related complications are identified and resolved. However, due to the high cost of such medication observance and its complexity, the surveillance is not considered a priority for this region is leaving the monitoring system feeble or absent.

Antibiotic resistance is a global challenge for public health and threatens health care system; thereby multifaceted interventions combined with solid political support from authorities are needed to tackle this global concern. Furthermore, enhancing patient and community education on the threat of antibiotic misuse and overuse and reinforcement of regulation and control of open access market to such medications must be imposed. Pharmacy students and health care facilities to provide an open access publications and educational writings to share the knowledge regarding the safe and efficient medication utilization and the hazards associated with overuse and possible misuse of drugs.

\section{Study Limitation and Future Research}

This study contains certain limitations that worth discussion. The article is based on observations, interpretations and discussion among the Kurdish community, the pharmacy students and professionals in the field of pharmaceutical industries and medical doctors in the region. Consequently, a larger-scale research study and statistical analysis is needed where various mix of population would define further the latitude of antibiotic use and misuse among Kurdish community and Kurdistan region.

The main result of this article is a foundation for the future interventions to enhance the awareness among Kurdish people and a wakeup call for the healthcare authorities in the region. To attain this, the author recommends several of the vital principles for controlling antibiotics overuse and subsequent antibiotic resistance.

\section{Conclusion and Recommendations}

The study indicates that irrational use of antibiotics in Kurdistan region is multifactor and threatens the society. Antibiotics are one of the most frequently retailed medications in the area as such anyone can visit a community pharmacy and purchase any pharmaceutical product as they would at a food market. The majority of the populations believe that antibiotics are effective for common cold and flu thus people consume by selfmedications. Doctors prescribe antimicrobial medications based on assumptions of bacterial infection rather than diagnostic assurance and most importantly to satisfy patients. Sanitary condition and inadequate surveillance of the imported medications are some of the major factors facing the region.

To combat such health threat, the author proposes some fundamental recommendations for controlling antibiotics misuse and subsequent antibiotic resistance. These include the followings:

- Develop an adult educational program regarding the conditions that require antibiotic treatments and when they are not necessary.

- Applying firm guidelines to control the channels of obtaining antibiotics and monitor quality of medications.

- Control the sale of antibiotics without prescription

- Assure patient compliance to antibiotic regimens as prescribed.

- Improved public sanitation and hygienic practices and develop a proper hospital infection control procedures.

- Develop a Rapid, low-cost, local laboratory facility for an antibiogram and antibiotic sensitivity test.

It is imperative to adopt strategies to warrant the implementation of these recommendations under current perplexing economic and political circumstances. It is well predicted by the present state of medical practice the antimicrobial resistances will be an important health issue in Kurdistan region and continue to worsen unless educative actions are instituted. 


\section{References}

Couper MR. (1997). The Rational Use of Antimicrobials, Clin. Infect. Dis. 1997;24 Suppl 1: S154-6.

Davies, S., Grant, J. and Catchpole, M. (2013). The drugs do not work. A Global Threat. London: Penguin Specials.

Department of Health (2012) Chief Medical Officer Annual Report 2011: Volume 2. Available at: http://www.gov.uk/government/publications/chief-medical-officerannual-report-volume-2

Dowell SF. (1998), Principles of judicious utilization of antibiotic agents for pediat-ric upper respiratory tract infections. Pediatrics 1998; 101(Suppl 1):163-84.

Edwards DJ, Eskin B. Richman PB, Mandell M, Bradley K, (2002) Parental use and misuse of antimicrobials: differences in urban vs. suburban settings. Acad Emerg Med 9: 22-26.

Goossens H, Elseviers M, Ferech M, Vander Stichele R, (2005) Outpatient antibiotic use in Europe and association with resistance: a cross-national database study. Lancet 365: 579-587.

Haak H, Hardon AP. (1988), Indigenised medications in developing countries: widely used, and neglected. Lancet 1988;2:620-1.

Hamm RM, Bemben DA., Hicks RJ, (1996), Antibiotics \& respiratory infections (patients more satisfied when expectations met), J Fam Pract 1996; 43:5662.

Huang WF, Wen KC, Hsiao ML. (1997), Adulteration thru synthetic therapeutic ingredients of traditional Chinese medicines in Taiwan. J Clin Pharmacol 1997;37:344-50.

Issack MI (2001), substandard drugs. Lancet 358: 1463.

Levy SB (2005) Antibiotic resistance-the problem intensifies. Adv. Drug Deliv. Rev 57: 1446-1450.

Llor C., Bjerrum L. (2014), Antibiotic resistance: risk associated with antimicrobial overuse and initiatives to reduce the problem. Advance Therapy in Drug Safety Vol. 5(6) 229 -241.

Llor, C., Butler, C. (2014), better also tests in primary care. Clin Infect Dis 58: 1487-1488.

MacFarlane J, MacFarlane R, Holmes W, Britten N. (1997), Influence of patients' expectations on the antimicrobial management of ALRT (acute lower respiratory tract) illness in general practice: questionnaire study. BMJ 1997; 315:12114.

Mainous AG, Zoorob RJ, Oler MJ, Haynes OM. (1997), Patient knowledge of upper respiratory infections: implications for antibiotic expectations and unnecessary utilization. J Fam Pract 1997;45(1):75-83.

McGregor A. (1997), Counterfeit drugs flood developing world. Lancet 1997;350:1690.

Meers PD. (1988), Infection control in developing countries. J. Hosp. Infect. 1988;11 Suppl A:406-10.

Okeke, Lamikanra, Edelman, (1999). Socioeconomic \& Behavioral Factors to Acquired Bacterial Resistance to Antibiotics. Emerging Infectious Diseases, Vol. 5, No. 1, January-February 1999.

Okello D, Lubanga R, Konde-Lule J, Arube-Wani J. (1997), Waste disposal in medical clinics in Kampala, Uganda. J. Clin. Epidemiology 1997;50 Suppl 1:45S.

Pearson CA. (1995), The role of district hospitals \& the action in international medicine network. Infect. Dis. Clin. North Am 1995;9:391-405.

SOCIO-ECONOMIC DETERMINANTS OF INCIDENCE OF TUBERCULOSIS IN .., Cornwall 1997 , http://www.theinternationaljournal.org/ojs/index.php?journal=tij\&page=articl (accessed January 20, 2016).

WHO (2012b), The evolving threat of antibiotic resistance, Options for action. Online: http://www.who.int/patientsafety/implementation/amr/publication/en/ Retrieved: 27 December 2015.

World Health Organization Report on Infectious Diseases (2000). Overcoming Microbial Resistance. Online: http://www.who.int/infectious-disease-report/2000/ Retrieved December 29, 2015. 